\title{
Glycerol as Precursor of Organoselanyl and Organotellanyl Alkynes
}

\author{
Eder J. Lenardão ${ }^{1, *}$, Elton L. Borges ${ }^{1}$, Guilherme Stach ${ }^{1}$, Liane K. Soares ${ }^{1}$, Diego Alves ${ }^{1}$, \\ Ricardo F. Schumacher ${ }^{1}$, Luana Bagnoli ${ }^{2}$, Francesca Marini ${ }^{2}$ and Gelson Perin ${ }^{1, *}$ \\ 1 Laboratório de Síntese Orgânica Limpa (LASOL), Centro de Ciências Químicas, Farmacêuticas e de \\ Alimentos (CCQFA), Universidade Federal de Pelotas (UFPel), P.O. Box 354, 96010-900 Pelotas, RS, Brazil; \\ chemistry_borges@yahoo.com.br (E.L.B.); guilherme.stach13@gmail.com (G.S.); \\ lianekrolowsoares@yahoo.com.br (L.K.S.); dsalves@gmail.com (D.A.); \\ ricardo.schumacher@ufpel.edu.br (R.F.S.) \\ 2 Department of Pharmaceutical Sciences, Group of Catalysis and Organic Green Chemistry, University of \\ Perugia, Via del Liceo 1, 06100 Perugia, Italy; luana.bagnoli@unipg.it (L.B.); francesca.marin i@unipg.it (F.M.) \\ * Correspondence: lenardao@ufpel.edu.br (E.J.L.); gelson_perin@ufpel.edu.br (G.P.); \\ Tel.: +55-53-3275-7533 (E.J.L. \& G.P.)
}

Academic Editor: Derek J. McPhee

Received: 24 January 2017; Accepted: 28 February 2017; Published: 2 March 2017

\begin{abstract}
Herein we describe the synthesis of organoselanyl and organotellanyl alkynes by the addition of lithium alkynylchalcogenolate (Se and Te) to tosyl solketal, easily obtained from glycerol. The alkynylchalcogenolate anions were generated in situ and added to tosyl solketal in short reaction times, furnishing in all cases the respective products of substitution in good yields. Some of the prepared compounds were deprotected using an acidic resin to afford new water-soluble 3-organotellanylpropane-1,2-diols. The synthetic versatility of the new chalcogenyl alkynes was demonstrated in the iodocyclization of 2,2-dimethyl-1,3dioxolanylmethyl(2-methoxyphenylethynyl)selane 3f, which afforded 3-iodo-2-(2,2-dimethyl-1,3dioxolanylmethyl) selenanylbenzo[ $b]$ furan in $85 \%$ yield, opening a new way to access water-soluble Se-functionalized benzo[b]furanes.
\end{abstract}

Keywords: selenium; tellurium; 1,3-dioxolanes; glycerol; alkynes

\section{Introduction}

Organochalcogen chemistry is considered a very broad field due to the increasing research on the synthesis [1-5] and applications [2,6-9] of this class of compounds. Besides, organoselanyl and organotellanyl alkynes have become extensively studied due their pharmacological and biological activities [10-13] and their use as starting material in organic synthesis [2,14-16]. For example, organotellanyl alkynes exhibited antidepressive-like activity [17], while alkyne-derived organotellanyl alkenes showed in vitro antioxidant activity with slight toxicity [18,19]. Additionally, chalcogenyl alkynes are useful in electrophilic cyclization reactions to prepare benzo[b]furans [20], in [4 + 2]-cycloadditions to produce the corresponding 2-chalcogenyl-1-halonaphthalenes [21], and in the synthesis of bis-phenylchalcogen alkenes [22,23]. Despite all the advances in this area, most of the papers are restricted to the synthesis of chalcogenyl alkynes starting mainly from aromatic terminal alkynes [24-30], with only a few methods to prepare aliphatic alkynylselenides and tellurides with different chalcogen substitution patterns.

On the other hand, with the increasing overproduction of glycerol [31], solketal has become a useful intermediate in chemical transformations using glycerol as a raw material. It is well accepted that tosyl solketal plays an important role in a vast array of applications [32-36]; standing out is its use 
as a building block in organic synthesis. Recently, tosyl solketal was used as starting material for the synthesis of several biologically active compounds [36,37]. The synthesis of a series of organochalcogen glycerol derivatives was described, including chalcogen ethers [38,39] with antioxidant activity [38], as well as enantiomerically pure selenides and diselenides [40].

In a previous paper [41], we described a convenient procedure for the synthesis of new vinyl chalcogenides by the reaction of glycerol-derived dichalcogenides with terminal alkynes in the presence of $\mathrm{NaBH}_{4}$, using PEG-400 as the solvent. Chalcogenyl alkynes were selectively prepared from the same starting materials, when ethanol was the solvent. However, reaction times were in the range of 5 to $26 \mathrm{~h}$, and the scope of the reaction was limited to organoselanyl alkynes, since the synthesis of only one organotellanyl alkyne in 55\% yield was reported [41]. Trying to solve these limitations, and in continuation of our studies on the synthesis and reactivity of chalcogenyl alkynes, herein we describe a general and efficient synthesis of a new glycerol-derived organoselanyl and organotellanyl alkyne 3 via the nucleophilic substitution of lithium alkynylchalcogenolate $\mathbf{1}^{\prime}$ (Se and Te) with tosyl solketal 2 (Scheme 1).

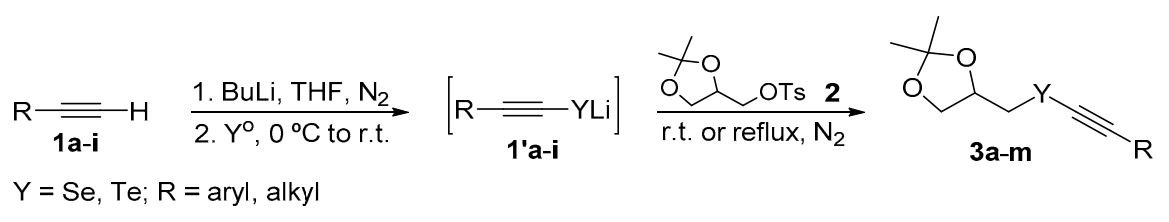

Scheme 1. General scheme of the reaction.

\section{Results and Discussion}

The first step of the reaction is the preparation of the nucleophilic species $\mathbf{1}^{\prime} \mathbf{a}-\mathbf{i}$, which were prepared in situ using a butyllithium solution and THF as the solvent. Phenylacetylene $1 \mathrm{a}(1.0 \mathrm{mmol})$ and elemental selenium $(1.0 \mathrm{mmol})$ were used as standard reagents to optimize the preparation of the respective alkynylselenolate $\mathbf{1}^{\prime} \mathbf{a}\left(\mathrm{R}=\mathrm{C}_{6} \mathrm{H}_{5} ; \mathrm{Y}=\mathrm{Se}\right)$ at $0{ }^{\circ} \mathrm{C}$ under $\mathrm{N}_{2}$ atmosphere. After stirring for $20 \mathrm{~min}$ at $0{ }^{\circ} \mathrm{C}$, racemic tosyl solketal $2(1.2 \mathrm{mmol})$ was added and the mixture was stirred at room temperature for an additional $1 \mathrm{~h}$, giving 2,2-dimethyl-1,3-dioxolanylmethyl(phenylethynyl)selane 3a in $20 \%$ yield. When the reaction time was extended to $3 \mathrm{~h}$, the yield was increased to $30 \%$, but side products were also observed. Next, the amount of tosyl solketal 2 was reduced to 1.0 mmol (1 equiv. related to the alkynylselenolate anion $\mathbf{1}^{\prime} \mathbf{a}$ ) and after $3 \mathrm{~h}$ at r.t., $3 \mathbf{3}$ was isolated in $50 \%$ yield. However, the best performance of this reaction was achieved when the amount of tosyl solketal 2 was decreased to $0.5 \mathrm{mmol}$ ( 0.5 equiv. related to $\mathbf{1}^{\prime} \mathbf{a}$ ), affording $3 \mathbf{a}$ in $80 \%$ yield after $3 \mathrm{~h}$. By using 0.7 equiv. of tosyl solketal 2, a decrease in the yield of product 3a was observed (42\%). These findings indicate that a large excess of chalcogenolate anion is mandatory for the reaction.

After determining the best conditions to prepare 3a, the protocol was extended to the differently substituted aliphatic and aromatic terminal alkynes $\mathbf{1 b} \mathbf{b} \mathbf{h}$ (Table 1, entries $\mathbf{2}-\mathbf{8})$. As shown in Table 1, a number of selenanyl alkynes were prepared in good yields. Starting from aliphatic hex-1-yne 1b, 2,2-dimethyl-1,3-dioxolanylmethyl(hex-1-yn-1-yl)selane $3 \mathbf{b}$ was obtained in $63 \%$ yield. This is a good outcome, considering that an aliphatic alkyne was used as the starting material (Table 1, entry 2). In principle, the presence of substituents in the aromatic ring of alkynes $1 \mathbf{c}-\mathbf{g}$ seems to negatively impact the reactivity, since all the respective products were obtained in lower yields. Experiments have shown that the overall result is similar in both situations; i.e., the yields of products $3 \mathbf{c}-\mathbf{g}$ are reduced to $60 \%-70 \%$ (Table 1, entries 3-7). Ethynylcyclohex-1-ene $\mathbf{1 h}$ afforded the respective selenanyl alkyne $3 \mathrm{~h}$ in $52 \%$ yield, showing that the reaction can be successfully applied to conjugated enynes (Table 1 , entry 8 ).

The optimized protocol was employed using tellurium instead selenium, in order to prepare

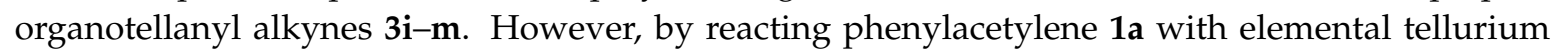
under the same reaction conditions employed for the selenium derivatives, the respective 
2,2-dimethyl-1,3-dioxolanylmethyl(phenylethynyl)tellane 3i was obtained in only 30\% yield after $3 \mathrm{~h}$. Trying to improve the yield of $\mathbf{3 i}$, the same procedure was repeated, but the tosyl solketal 2 was added at $0{ }^{\circ} \mathrm{C}$ and the temperature maintained at $0{ }^{\circ} \mathrm{C}$ for an additional $3 \mathrm{~h}$. In this case, the desired product $3 \mathrm{~h}$ was obtained only in trace amounts, with a large amount of the bis(phenylethynyl)tellane as side product. Then, an additional test was performed: after addition of the solketal 2 to the previously formed alkynyltellurolate $\mathbf{1}^{\prime}$ at $0{ }^{\circ} \mathrm{C}$, the ice bath was replaced by an oil bath and the reaction mixture was stirred under reflux for $1.5 \mathrm{~h}$. To our delight, this reaction afforded the desired tellanyl alkyne $3 \mathbf{i}$ in $85 \%$ yield (Table 1, entry 9). Under the new conditions, the reaction scope could be expanded to other alkynes and the respective tellanyl alkynes were obtained in good yields, similar to the selanyl alkynes analogues (Table 1, entries 10-13).

Table 1. Scope of the synthesis of organoselanyl $3 \mathbf{a}-\mathbf{h}$ and organotellanyl alkynes $3 \mathbf{i}-\mathbf{m}^{\mathrm{a}}$.

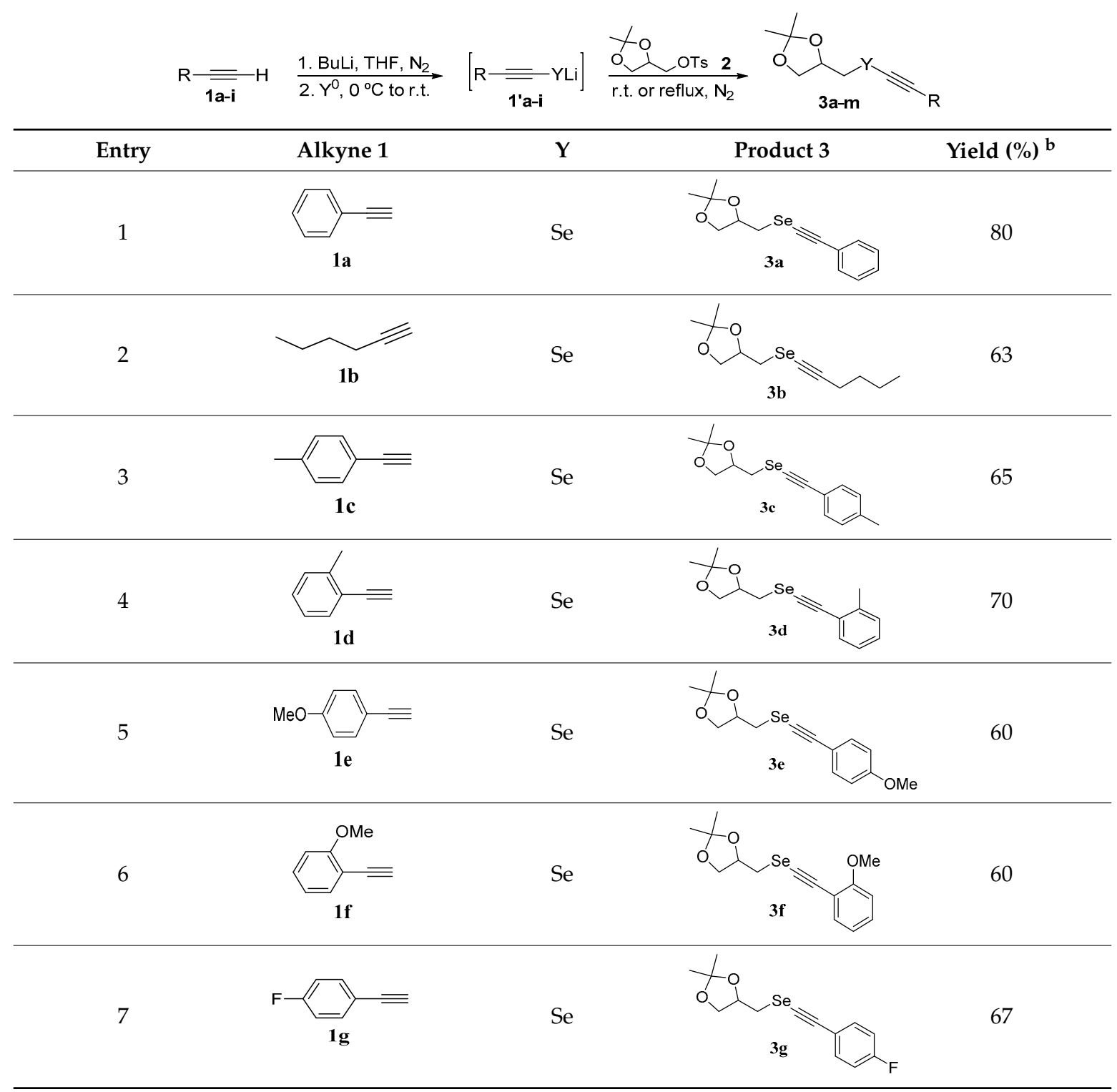


Table 1. Cont.

\begin{tabular}{|c|c|c|c|c|}
\hline Entry & Alkyne 1 & $\mathbf{Y}$ & Product 3 & Yield (\%) ${ }^{b}$ \\
\hline 8 & $1 \mathrm{~h}$ & Se & $3 \mathrm{~h}$ & 52 \\
\hline 9 & $1 a$ & $\mathrm{Te}$ & & 85 \\
\hline 10 & $1 b$ & $\mathrm{Te}$ & & 63 \\
\hline 11 & 1c & $\mathrm{Te}$ & & 55 \\
\hline 12 & 1h & $\mathrm{Te}$ & & 61 \\
\hline 13 & & $\mathrm{Te}$ & $3 m$ & 63 \\
\hline
\end{tabular}

a Reaction was performed using alkyne $1(1.0 \mathrm{mmol}), \mathrm{Se}^{0}$ or $\mathrm{Te}^{0}(1.0 \mathrm{mmol})$, butyllithium solution (1.6 mol/L in hexanes; $1.0 \mathrm{mmol})$ at $0{ }^{\circ} \mathrm{C}$ in THF $(5.0 \mathrm{~mL})$ under $\mathrm{N}_{2}$ atmosphere. Then, tosyl solketal $2(0.5 \mathrm{mmol})$ in THF $(2.0 \mathrm{~mL})$ was added at r.t. and the mixture stirred for additional $3 \mathrm{~h}(\mathbf{3 a}-\mathbf{h})$ or under reflux for $1.5 \mathrm{~h}(3 \mathbf{i}-\mathbf{m})$; ${ }^{b}$ Yields are given for isolated products.

Due to our interest in the synthetic applications of organochalcogen compounds, as well as in studying new possibilities in their biological activities, we performed the synthesis of new 3-organotellanylpropane-1,2-diols 4 (Table 2). Starting from the organotellanyl alkynes 3i, 31, and $3 \mathrm{~m}$, the 3-tellanylpropane-1,2-diols $4 \mathbf{a}-\mathrm{c}$ were obtained in moderate yields by treatment with Dowex 50WX8 ( $\mathrm{H}^{+}$form) in methanol, after $5 \mathrm{~h}$ (Table 2, entries 1-3). These new compounds generally showed a good solubility in water, which will facilitate biological tests where aqueous medium is required (Table 2).

Selanyl alkynes with an appropriate substitution pattern are attractive intermediates in organic synthesis [20]. For example, the intramolecular electrophilic cyclization of $\mathbf{3 f}$ with iodine was easily conduced in $\mathrm{CH}_{2} \mathrm{Cl}_{2}$ at r.t. for $1 \mathrm{~h}$, delivering $85 \%$ yield of the densely functionalized 3-iodo-2-(2,2-dimethyl-1,3-dioxolanylmethyl)selenanylbenzo[b]furan 5 (Scheme 2). The combination of a selenium group with the benzo[b]furan scaffold could be an interesting strategy in the prospection of new drug candidates. 
Table 2. Synthesis of new 3-organotellanylpropane-1,2-diols $4 a-c^{a}$.

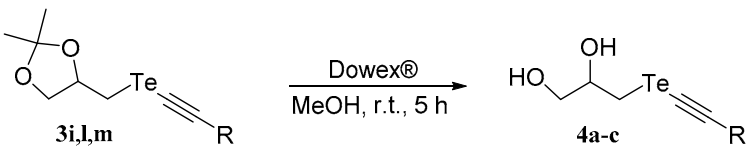

Entry

${ }^{\mathrm{a}}$ Reaction was performed using $1.0 \mathrm{mmol}$ of $3,1.112 \mathrm{~g}$ of Dowex ${ }^{\circledR}$ in $2.5 \mathrm{~mL}$ of $\mathrm{MeOH}$ at r.t. for $5 \mathrm{~h}^{\mathrm{b}}{ }^{\mathrm{b}}$ Solubility measured in water; ${ }^{\mathrm{c}}$ Yields are given for isolated products.
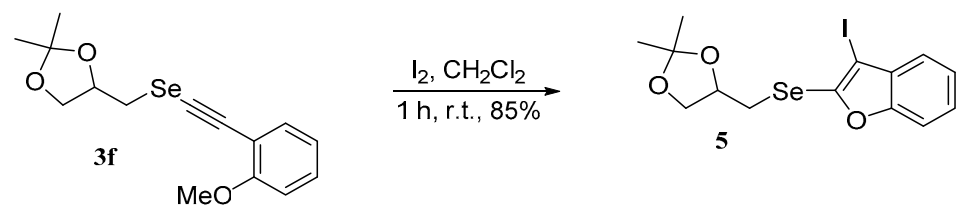

Scheme 2. Synthesis of 3-iodo-2-(2,2-dimethyl-1,3-dioxolanylmethyl)selenanylbenzo[b]furan 5.

\section{Experimental Section}

\subsection{General Information}

The reactions were monitored by thin layer chromatography (TLC) carried out on Merck (Merck, Darmstadt, Germany) silica gel $\left(60 \mathrm{~F}_{254}\right)$ by using UV light as visualizing agent and 5\% vanillin in $10 \%$ $\mathrm{H}_{2} \mathrm{SO}_{4}$ and heat as developing agent. NMR spectra were recorded with Bruker spectrometer (Bruker, Billerica, MA, USA) DPX 300, DPX 400, and DPX 500 (300, 400, and $500 \mathrm{MHz}$, respectively) instruments using $\mathrm{CDCl}_{3}$ as solvent and calibrated using tetramethylsilane (TMS) as internal standard. Chemical shifts $(\delta)$ are reported in ppm, coupling constants $(J)$ are reported in Hertz. The NMR spectra are found in the Supplementary Materials. Low-resolution mass spectra were obtained with a Shimadzu GC-MS-QP2010 mass spectrometer (Shimadzu Corporation, Kyoto, Japan) and molecular ion values are reported according the exact mass. High-resolution mass spectra (HMRS) were recorded on a Shimadzu LC-MS-IT-TOF spectrometer (Shimadzu Corporation). Melting points were determined using a Marte PFD III melting point instrument (Marte Científica, Minas Gerais, Brazil).

\subsection{General Procedure for Synthesis of Organoselanyl Alkynes 3a-h}

To a solution of alkyne $1(1.0 \mathrm{mmol})$ in THF $(5.0 \mathrm{~mL})$ under $\mathrm{N}_{2}$ atmosphere, BuLi $(1.6 \mathrm{~mol} / \mathrm{L}$ in hexanes; $1.0 \mathrm{mmol}$ ) was added at $0{ }^{\circ} \mathrm{C}$. After $20 \mathrm{~min}$, the temperature was increased to room temperature, and elemental selenium $\left(\mathrm{Se}^{0}, 1.0 \mathrm{mmol}\right)$ was added. The stirring at room temperature was maintained until all selenium was consumed, and then a solution of racemic tosyl solketal 2 $(0.5 \mathrm{mmol})$ in THF $(2.0 \mathrm{~mL})$ was added. After stirring for $3 \mathrm{~h}$, the reaction mixture was quenched with 
water $(15.0 \mathrm{~mL})$ and extracted with ethyl acetate $(3 \times 15.0 \mathrm{~mL})$. The organic phase was separated, dried over $\mathrm{MgSO}_{4}$, and the solvent was evaporated under reduced pressure. The product was isolated by column chromatography using hexanes/ethyl acetate as eluent.

Analytical Data of Products 3a-h

2,2-Dimethyl-1,3-dioxolanylmethyl(phenylethynyl)selane 3a (Table 1, entry 1) [41]: Yield: $0.118 \mathrm{~g}(80 \%)$; yellow oil. ${ }^{1} \mathrm{H}-\mathrm{NMR}\left(\mathrm{CDCl}_{3}, 300 \mathrm{MHz}\right) ; \delta(\mathrm{ppm}): 7.38-7.42(\mathrm{~m}, 2 \mathrm{H}, \mathrm{Ar}-\mathrm{H}), 7.27-7.32(\mathrm{~m}, 3 \mathrm{H}, \mathrm{Ar}-\mathrm{H})$, $4.46-4.54(\mathrm{~m}, 1 \mathrm{H}, \mathrm{O}-\mathrm{CH}), 4.21(\mathrm{dd}, J=8.6$ and $6.0 \mathrm{~Hz}, 1 \mathrm{H}, \mathrm{O}-\underline{\mathrm{HCH}}), 3.88(\mathrm{dd}, J=8.6$ and $5.8 \mathrm{~Hz}, 1 \mathrm{H}$, O- $\underline{\mathrm{HCH}}), 3.07(\mathrm{dd}, J=12.1$ and $5.2 \mathrm{~Hz}, 1 \mathrm{H}, \mathrm{Se}-\underline{\mathrm{HCH}}), 2.94(\mathrm{dd}, J=12.1$ and $7.7 \mathrm{~Hz}, 1 \mathrm{H}, \mathrm{Se}-\underline{\mathrm{HCH}}), 1.45$ $\left(\mathrm{d}, J=0.5 \mathrm{~Hz}, 3 \mathrm{H}, \mathrm{C}-\mathrm{CH}_{3}\right), 1.37\left(\mathrm{~d}, J=0.5 \mathrm{~Hz}, \mathrm{C}-\mathrm{CH}_{3}\right) .{ }^{13} \mathrm{C}-\mathrm{NMR}\left(\mathrm{CDCl}_{3}, 75 \mathrm{MHz}\right) ; \delta(\mathrm{ppm}): 131.5$, 128.3 (3C), 123.2, 109.7, 99.4, 75.3, 69.3, 68.9, 31.8, 27.0, 25.5. MS: $m / z$ (rel. int.) 296 (M+ , 8.7), 181 (43.8), 115 (25.9), 102 (26.4), 43 (100.0).

2,2-Dimethyl-1,3-dioxolanylmethyl(hex-1-yn-1-yl)selane 3b (Table 1, entry 2) [41]: Yield: $0.087 \mathrm{~g}(63 \%)$; yellow oil. MS: $m / z$ (rel. int.) $276\left(\mathrm{M}^{+}, 8.5\right), 101$ (39.1), 79 (22.4), 57 (31.0), 43 (100.0).

2,2-Dimethyl-1,3-dioxolanylmethyl(4-methylphenylethynyl)selane 3c (Table 1, entry 3) [41]: Yield: $0.101 \mathrm{~g}$ (65\%); yellow solid. m.p. $45-47^{\circ} \mathrm{C}$. MS: $\mathrm{m} / \mathrm{z}$ (rel. int.) $310\left(\mathrm{M}^{+}, 23.7\right), 195$ (66.0), 115 (87.0), 57 (66.0), $43(100.0)$.

2,2-Dimethyl-1,3-dioxolanylmethyl(2-methylphenylethynyl)selane 3d (Table 1, entry 4) [41]: Yield: $0.109 \mathrm{~g}$ (70\%); yellow oil. MS: $m / z$ (rel. int.) $310\left(\mathrm{M}^{+}, 9.3\right), 195$ (12.4), 115 (100.0), 101 (10.0), 43 (39.7).

2,2-Dimethyl-1,3-dioxolanylmethyl(4-methoxyphenylethynyl)selane 3e (Table 1, entry 5) [41]: Yield: $0.098 \mathrm{~g}$ (60\%); yellow solid; m.p. $39-41{ }^{\circ} \mathrm{C} .{ }^{1} \mathrm{H}-\mathrm{NMR}\left(\mathrm{CDCl}_{3}, 400 \mathrm{MHz}\right) ; \delta(\mathrm{ppm}): 7.27(\mathrm{~d}, J=8.9 \mathrm{~Hz}, 2 \mathrm{H}, \mathrm{Ar}-\mathrm{H})$, $6.74(\mathrm{~d}, J=8.9 \mathrm{~Hz}, 2 \mathrm{H}, \mathrm{Ar}-\mathrm{H}), 4.37-4.43(\mathrm{~m}, 1 \mathrm{H}, \mathrm{O}-\mathrm{CH}), 4.12(\mathrm{dd}, J=8.5$ and $6.0 \mathrm{~Hz}, 1 \mathrm{H}, \mathrm{O}-\underline{\mathrm{HCH}}), 3.79$ $(\mathrm{dd}, J=8.5$ and $5.9 \mathrm{~Hz}, 1 \mathrm{H}, \mathrm{O}-\underline{\mathrm{HCH}}), 3.71\left(\mathrm{~s}, 3 \mathrm{H}, \mathrm{Ar}-\mathrm{OCH}_{3}\right), 2.98(\mathrm{dd}, J=12.1$ and $5.1 \mathrm{~Hz}, 1 \mathrm{H}, \mathrm{Se}-\underline{\mathrm{HCH}})$, $2.84(\mathrm{dd}, J=12.1$ and $7.7 \mathrm{~Hz}, 1 \mathrm{H}, \mathrm{Se}-\underline{\mathrm{HCH}}), 1.37\left(\mathrm{~s}, 3 \mathrm{H}, \mathrm{C}-\mathrm{CH}_{3}\right), 1.29\left(\mathrm{~s}, 3 \mathrm{H}, \mathrm{C}-\mathrm{CH}_{3}\right) .{ }^{13} \mathrm{C}-\mathrm{NMR}\left(\mathrm{CDCl}_{3}\right.$, $100 \mathrm{MHz}) ; \delta$ (ppm): 159.8, 133.3, 115.5, 113.9, 109.7, 99.3, 75.4, 69.0, 67.3, 55.2, 31.8, 27.0, 25.5. MS: $\mathrm{m} / \mathrm{z}$ (rel. int.) $326\left(\mathrm{M}^{+}, 3.4\right), 211$ (39.3), 196 (20.2), 132 (57.1), 43 (100.0).

2,2-Dimethyl-1,3-dioxolanylmethyl(2-methoxyphenylethynyl)selane 3f (Table 1, entry 6): Yield: $0.098 \mathrm{~g}$ (60\%); yellow oil. ${ }^{1} \mathrm{H}-\mathrm{NMR}\left(\mathrm{CDCl}_{3}, 400 \mathrm{MHz}\right) ; \delta(\mathrm{ppm}): 7.35-7.37(\mathrm{~m}, 1 \mathrm{H}, \mathrm{Ar}-\mathrm{H}), 7.24-7.28(\mathrm{~m}, 1 \mathrm{H}$, Ar-H), 6.84-6.90 (m, 2H, Ar-H), 4.50-4.56 (m, 1H, O-CH), 4.21-4.25 (m, 1H, O- $\underline{\mathrm{HCH}}), 3.93$ (dd, J = 7.7 and $6.1 \mathrm{~Hz}, 1 \mathrm{H}, \mathrm{O}-\underline{\mathrm{HCH}}), 3.86\left(\mathrm{~s}, 3 \mathrm{H}, \mathrm{Ar}-\mathrm{OCH}_{3}\right), 3.08(\mathrm{dd}, J=12.0$ and $4.0 \mathrm{~Hz}, 1 \mathrm{H}, \mathrm{Se}-\underline{\mathrm{HCH}}), 2.93$ $(\mathrm{dd}, J=12.0$ and $8.0 \mathrm{~Hz}, 1 \mathrm{H}$, Se- $\underline{\mathrm{HCH}}), 1.45\left(\mathrm{~s}, 3 \mathrm{H}, \mathrm{C}-\mathrm{CH}_{3}\right), 1.37\left(\mathrm{~s}, 3 \mathrm{H}, \mathrm{C}-\mathrm{CH}_{3}\right) .{ }^{13} \mathrm{C}-\mathrm{NMR}\left(\mathrm{CDCl}_{3}\right.$, $100 \mathrm{MHz}$ ); $\delta$ (ppm): 160.1, 133.3, 129.6, 120.3, 112.4, 110.5, 109.5, 95.7, 75.5, 73.0, 69.0, 55.6, 31.7, 26.9, 25.5. MS: $m / z$ (rel. int.) $326\left(\mathrm{M}^{+}, 29.4\right), 131$ (100.0), 119 (51.2), 57 (71.9), 43 (91.4). HRMS: Calculated mass to $\mathrm{C}_{15} \mathrm{H}_{18} \mathrm{O}_{3} \mathrm{Se}:[\mathrm{M}]^{+} 326.0421$, found: 326.0439 .

2,2-Dimethyl-1,3-dioxolanylmethyl(4-fluorophenylethynyl)selane 3g (Table 1, entry 7) [41]: Yield: $0.105 \mathrm{~g}$ (67\%); yellow solid; m.p. 37-39 ${ }^{\circ} \mathrm{C}$. MS: $\mathrm{m} / z$ (rel. int.) $314\left(\mathrm{M}^{+}, 0.3\right), 199$ (32.2), 120 (27.1), 107 (90.6), $43(100.0)$.

2,2-Dimethyl-1,3-dioxolanylmethyl(cyclohex-1-en-1-ylethynyl)selane 3h (Table 1, entry 8): Yield: $0.078 \mathrm{~g}$ (52\%); yellow oil. ${ }^{1} \mathrm{H}-\mathrm{NMR}\left(\mathrm{CDCl}_{3}, 400 \mathrm{MHz}\right) ; \delta$ (ppm): 6.07-6.09 (m, 1H, C=CH), 4.41-4.45 (m, 1H, $\mathrm{O}-\mathrm{CH}), 4.18(\mathrm{dd}, J=8.5$ and $6.0 \mathrm{~Hz}, 1 \mathrm{H}, \mathrm{O}-\underline{\mathrm{HCH}}), 3.83(\mathrm{dd}, J=8.5$ and $5.9 \mathrm{~Hz}, 1 \mathrm{H}, \mathrm{O}-\underline{\mathrm{HCH}}), 2.99$ $(\mathrm{dd}, J=12.1$ and $5.0 \mathrm{~Hz}, 1 \mathrm{H}, \mathrm{Se}-\underline{\mathrm{HCH}}), 2.84(\mathrm{dd}, J=12.1$ and $8.0 \mathrm{~Hz}, 1 \mathrm{H}, \mathrm{Se}-\underline{\mathrm{HCH}}), 2.09-2.10(\mathrm{~m}, 4 \mathrm{H})$, $1.55-1.65(\mathrm{~m}, 4 \mathrm{H}), 1.43\left(\mathrm{~s}, 3 \mathrm{H}, \mathrm{C}-\mathrm{CH}_{3}\right), 1.36\left(\mathrm{~s}, 3 \mathrm{H}, \mathrm{C}-\mathrm{CH}_{3}\right) .{ }^{13} \mathrm{C}-\mathrm{NMR}\left(\mathrm{CDCl}_{3}, 100 \mathrm{MHz}\right) ; \delta(\mathrm{ppm}): 135.3$, 121.0, 109.6, 101.5, 75.5, 69.0, 65.7, 31.6, 29.1, 27.0, 25.61, 25.56, 22.3, 21.4. MS: $\mathrm{m} / z$ (rel. int.) $300\left(\mathrm{M}^{+}, 6.0\right)$, 185 (6.7), 104 (35.1), 91 (19.9), 43 (100.0). HRMS: Calculated mass to $\mathrm{C}_{14} \mathrm{H}_{20} \mathrm{O}_{2} \mathrm{Se}:[\mathrm{M}+\mathrm{H}]^{+}$301.0707, found: 301.0690 . 


\subsection{General Procedure for the Synthesis of Organotellanyl Alkynes 3i-m}

To a solution of alkyne $1(1.0 \mathrm{mmol})$ in THF $(5.0 \mathrm{~mL})$ under $\mathrm{N}_{2}$ atmosphere, BuLi $(1.6 \mathrm{~mol} / \mathrm{L}$ in hexanes; $1.0 \mathrm{mmol}$ ) was added at $0{ }^{\circ} \mathrm{C}$. After $20 \mathrm{~min}$, the temperature was increased to room temperature and elemental tellurium $\left(\mathrm{Te}^{0}, 1.0 \mathrm{mmol}\right)$ was added. The stirring at room temperature was maintained until all tellurium has been consumed and then a solution of racemic tosyl solketal $2(0.5 \mathrm{mmol})$ in THF $(2.0 \mathrm{~mL})$ was added and the mixture was stirred under reflux for additional $1.5 \mathrm{~h}$. After, the reaction mixture was quenched with water $(15.0 \mathrm{~mL})$ and extracted with ethyl acetate $(3 \times 15.0 \mathrm{~mL})$. The organic phase was separated, dried over $\mathrm{MgSO}_{4}$, and the solvent was evaporated under reduced pressure. The product was isolated by column chromatography using hexanes/ethyl acetate as eluent.

\section{Analytical Data of Products 3i-m}

2,2-Dimethyl-1,3-dioxolanylmethyl(phenylethynyl)tellane 3i (Table 1, entry 9) [41]: Yield: $0.147 \mathrm{~g}(85 \%)$; red oil. ${ }^{1} \mathrm{H}-\mathrm{NMR}\left(\mathrm{CDCl}_{3}, 400 \mathrm{MHz}\right) ; \delta$ (ppm): 7.31-7.34 (m, 2H, Ar-H), 7.18-7.25 (m, 3H, Ar-H), 4.38-4.44 $(\mathrm{m}, 1 \mathrm{H}, \mathrm{O}-\mathrm{CH}), 4.12(\mathrm{dd}, J=8.4$ and $6.1 \mathrm{~Hz}, 1 \mathrm{H}, \mathrm{O}-\underline{\mathrm{HCH}}), 3.73(\mathrm{dd}, J=8.4$ and $6.1 \mathrm{~Hz}, 1 \mathrm{H}, \mathrm{O}-\underline{\mathrm{HCH}})$, 3.00-3.08 (m, 2H, Te-CH $), 1.37\left(\mathrm{~s}, 3 \mathrm{H}, \mathrm{C}-\mathrm{CH}_{3}\right), 1.28\left(\mathrm{~s}, 3 \mathrm{H}, \mathrm{C}-\mathrm{CH}_{3}\right) .{ }^{13} \mathrm{C}-\mathrm{NMR}\left(\mathrm{CDCl}_{3}, 100 \mathrm{MHz}\right) ; \delta$ (ppm): 131.7, 128.3, 128.2, 123.4, 111.3, 109.7, 75.9, 70.0, 44.2, 27.0, 25.6, 13.6. MS: $m / z$ (rel. int.) 346 (M+, 32.7), 231 (58.2), 101 (75.7), 57 (100.0), 43 (77.0).

2,2-Dimethyl-1,3-dioxolanylmethyl(hex-1-yn-1-yl)tellane 3j (Table 1, entry 10): Yield: $0.103 \mathrm{~g}$ (63\%); red oil. ${ }^{1} \mathrm{H}-\mathrm{NMR}\left(\mathrm{CDCl}_{3}, 500 \mathrm{MHz}\right) ; \delta(\mathrm{ppm}): 4.41-4.46(\mathrm{~m}, 1 \mathrm{H}, \mathrm{O}-\mathrm{CH}), 4.18(\mathrm{dd}, J=8.4$ and $6.0 \mathrm{~Hz}, 1 \mathrm{H}$, $\mathrm{O}-\underline{\mathrm{HCH}}), 3.77(\mathrm{dd}, J=8.4$ and $6.4 \mathrm{~Hz}, 1 \mathrm{H}, \mathrm{O}-\underline{\mathrm{HCH}}), 3.02(\mathrm{dd}, J=11.7$ and $5.2 \mathrm{~Hz}, 1 \mathrm{H}, \mathrm{Te}-\underline{\mathrm{HCH}}), 2.97$ $(\mathrm{dd}, J=11.7$ and $7.6 \mathrm{~Hz}, 1 \mathrm{H}, \mathrm{Te}-\underline{\mathrm{HCH}}), 2.48\left(\mathrm{t}, J=7.0 \mathrm{~Hz}, 2 \mathrm{H}, \mathrm{CH}_{2} \mathrm{C}_{\mathrm{sp}}\right), 1.47-1.53(\mathrm{~m}, 2 \mathrm{H}), 1.43(\mathrm{~s}, 3 \mathrm{H}$, $\left.\mathrm{C}-\mathrm{CH}_{3}\right), 1.37-1.43(\mathrm{~m}, 2 \mathrm{H}), 1.35\left(\mathrm{~s}, 3 \mathrm{H}, \mathrm{C}-\mathrm{CH}_{3}\right), 0.91(\mathrm{t}, J=7.3 \mathrm{~Hz}, 3 \mathrm{H}) .{ }^{13} \mathrm{C}-\mathrm{NMR}\left(\mathrm{CDCl}_{3}, 125 \mathrm{MHz}\right) ; \delta$ (ppm): 112.8, 109.7, 76.2, 70.0, 31.0, 27.0, 25.6, 21.9, 20.6, 13.5, 12.4. MS: $m / z$ (rel. int.) $326\left(\mathrm{M}^{+}, 21.5\right), 115$ (63.0), 81 (79.9), 57 (100.0), 43 (77.2). HRMS: Calculated mass for $\mathrm{C}_{12} \mathrm{H}_{20} \mathrm{O}_{2} \mathrm{Te}:[\mathrm{M}+\mathrm{OH}]^{+} 343.0553$, found: 343.0533 .

2,2-Dimethyl-1,3-dioxolanylmethyl(4-methylphenylethynyl)tellane 3k (Table 1, entry 11): Yield: $0.099 \mathrm{~g}$ (55\%); red oil. ${ }^{1} \mathrm{H}-\mathrm{NMR}\left(\mathrm{CDCl}_{3}, 500 \mathrm{MHz}\right) ; \delta(\mathrm{ppm}): 7.29(\mathrm{~d}, J=8.1 \mathrm{~Hz}, 2 \mathrm{H}, \mathrm{Ar}-\mathrm{H}), 7.10(\mathrm{~d}, J=8.1 \mathrm{~Hz}$, 2H, Ar-H), 4.45-4.50 (m, 1H, O-CH), $4.20(\mathrm{dd}, J=8.4$ and $6.0 \mathrm{~Hz}, 1 \mathrm{H}, \mathrm{O}-\underline{\mathrm{HCH}}), 3.81(\mathrm{dd}, J=8.4$ and $6.4 \mathrm{~Hz}, 1 \mathrm{H}, \mathrm{O}-\underline{\mathrm{HCH}}), 3.10-3.11$ (m, 2H, Te-CH$), 2.35$ (s, 3H, Ar-CH $\left.\mathrm{CH}_{3}\right), 1.45\left(\mathrm{~s}, 3 \mathrm{H}, \mathrm{C}-\mathrm{CH}_{3}\right), 1.35$ (s, $3 \mathrm{H}$, $\left.\mathrm{C}-\mathrm{CH}_{3}\right) .{ }^{13} \mathrm{C}-\mathrm{NMR}\left(\mathrm{CDCl}_{3}, 125 \mathrm{MHz}\right) ; \delta(\mathrm{ppm}):$ 138.6, 131.7, 128.9, 120.4, 111.4, 109.7, 76.0, 70.0, 42.9, 27.0, 25.6, 21.4, 13.5. MS: $m / z$ (rel. int.) 360 (M+, 12.8), 245 (27.8), 115 (100.0), 57 (78.9), 43 (75.2). HRMS: Calculated mass for $\mathrm{C}_{15} \mathrm{H}_{18} \mathrm{O}_{2} \mathrm{Te}:[\mathrm{M}+\mathrm{H}]^{+}$361.0447, found: 361.0469 .

2,2-Dimethyl-1,3-dioxolanylmethyl(cyclohex-1-en-1-ylethynyl)tellane 31 (Table 1, entry 12): Yield: $0.107 \mathrm{~g}$ (61\%); yellow oil; ${ }^{1} \mathrm{H}-\mathrm{NMR}\left(\mathrm{CDCl}_{3}, 500 \mathrm{MHz}\right) ; \delta(\mathrm{ppm})$ : 6.06-6.09 (m, 1H, C=CH), 4.42-4.47 (m, $1 \mathrm{H}, \mathrm{O}-\mathrm{CH}), 4.19(\mathrm{dd}, J=8.4$ and $5.9 \mathrm{~Hz}, 1 \mathrm{H}, \mathrm{O}-\underline{\mathrm{HCH}}), 3.78(\mathrm{dd}, \mathrm{J}=8.4$ and $6.4 \mathrm{~Hz}, 1 \mathrm{H}, \mathrm{O}-\underline{\mathrm{HCH}})$, 3.02-3.03 (m, 2H, Te-CH $), 2.08-2.16(\mathrm{~m}, 4 \mathrm{H}), 1.54-1.65(\mathrm{~m}, 4 \mathrm{H}), 1.44\left(\mathrm{~s}, 3 \mathrm{H}, \mathrm{C}-\mathrm{CH}_{3}\right), 1.35(\mathrm{~s}, 3 \mathrm{H}, \mathrm{C}-\mathrm{CH})$. ${ }^{13} \mathrm{C}-\mathrm{NMR}\left(\mathrm{CDCl}_{3}, 125 \mathrm{MHz}\right) ; \delta$ (ppm): 135.9, 121.1, 113.5, 109.7, 76.2, 70.0, 39.9, 29.2, 27.0, 25.6, 25.5, 22.2, 21.4, 13.2. MS: $m / z$ (rel. int.) $350\left(\mathrm{M}^{+}, 40.3\right), 115$ (31.8), 105 (100.0), 57 (92.9), 43 (73.8). HRMS: Calculated mass for $\mathrm{C}_{14} \mathrm{H}_{20} \mathrm{O}_{2} \mathrm{Te}:[\mathrm{M}+\mathrm{H}]^{+}$351.0604, found: 351.0597 .

2,2-Dimethyl-1,3-dioxolanylmethyl(4-tert-buthylphenylethynyl)tellane 3m (Table 1, entry 13): Yield: $0.127 \mathrm{~g}$ (63\%); red oil. ${ }^{1} \mathrm{H}$ NMR $\left(\mathrm{CDCl}_{3}, 500 \mathrm{MHz}\right) ; \delta(\mathrm{ppm}): 7.31-7.35$ (m, 4H, Ar-H), 4.46-4.51 (m, 1H, O-CH), $4.21(\mathrm{dd}, J=8.4$ and $6.0 \mathrm{~Hz}, 1 \mathrm{H}, \mathrm{O}-\underline{\mathrm{HCH}}), 3.81(\mathrm{dd}, J=8.4$ and $6.4 \mathrm{~Hz}, 1 \mathrm{H}, \mathrm{O}-\underline{\mathrm{HCH}}), 3.10-3.11(\mathrm{~m}, 2 \mathrm{H}$,

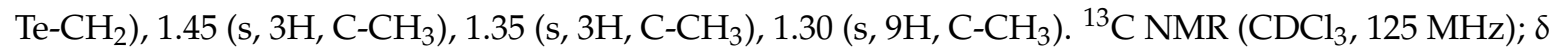
(ppm): 151.7, 131.6, 125.2, 120.4, 111.5, 109.7, 76.1, 70.0, 42.9, 34.7, 31.1, 27.0, 25.6, 13.5. MS: $\mathrm{m} / z$ (rel. int.) 402 ( $\mathrm{M}^{+}$, 26.2), 287 (19.6), 143 (66.4), 57 (99.7), 43 (100.0). HRMS: Calculated mass for $\mathrm{C}_{18} \mathrm{H}_{24} \mathrm{O}_{2} \mathrm{Te}$ : $[\mathrm{M}+\mathrm{H}]^{+}$403.0917, found: 403.0908 . 


\subsection{General Procedure for the Synthesis of 3-(Organotellanyl)propane-1,2-diol 4a-c}

To a solution of the respective organotellanyl alkyne $3(1.0 \mathrm{mmol})$ in $\mathrm{MeOH}(2.5 \mathrm{~mL})$ Dowex ${ }^{\circledR}$ acidic ion-exchange resin (50WX8 20-50 mesh; $1.122 \mathrm{~g}$ ) was added at room temperature. The reaction mixture was stirred for $5 \mathrm{~h}$ at room temperature and then it was filtered and washed with $\mathrm{MeOH}$. The filtrate was concentrated and chromatographed (50\% EtOAc/hexanes).

Analytical Data of Products $4 \mathbf{a}-\mathbf{c}$

3-(Phenylethynyltellanyl)propane-1,2-diol 4a (Table 2, entry 1): Yield: $0.153 \mathrm{~g}$ (50\%); red oil. ${ }^{1} \mathrm{H}-\mathrm{NMR}$ $\left(\mathrm{CDCl}_{3}, 400 \mathrm{MHz}\right) ; \delta$ (ppm): 7.39-7.41 (m, 2H, Ar-H), 7.26-7.32 (m, 3H, Ar-H), 4.04-4.09 (m, 1H, O-CH), $3.81(\mathrm{dd}, J=11.3$ and $3.1 \mathrm{~Hz}, 1 \mathrm{H}, \mathrm{O}-\underline{\mathrm{HCH}}), 3.66(\mathrm{dd}, J=11.3$ and $6.1 \mathrm{~Hz}, 1 \mathrm{H}, \mathrm{O}-\underline{\mathrm{HCH}}), 2.81-3.07(\mathrm{~m}$, $4 \mathrm{H}, \mathrm{Te}-\mathrm{CH}_{2}$ and $\left.2 \mathrm{O}-\mathrm{H}\right) .{ }^{13} \mathrm{C}-\mathrm{NMR}\left(\mathrm{CDCl}_{3}, 100 \mathrm{MHz}\right) ; \delta(\mathrm{ppm}): 131.8,128.4,128.2,123.4,111.2,71.5$, 66.6, 45.1, 14.8. MS: $m / z$ (rel. int.) $306\left(\mathrm{M}^{+}, 7.9\right), 231$ (11.7), 155 (44.9), 102 (43.7), 91 (100.0). HRMS: Calculated mass for $\mathrm{C}_{11} \mathrm{H}_{12} \mathrm{O}_{2} \mathrm{Te}:[\mathrm{M}+\mathrm{H}]^{+}$306.9978, found: 306.9903 .

3-(Cyclohex-1-en-1-ylethynyltellanyl)propane-1,2-diol 4b (Table 2, entry 2): Yield: $0.155 \mathrm{~g}$ (50\%); yellow oil. ${ }^{1} \mathrm{H}-\mathrm{NMR}\left(\mathrm{CDCl}_{3}, 400 \mathrm{MHz}\right) ; \delta$ (ppm): 6.07-6.10 (m, 1H, C=CH), 4.0-4.06 (m, 1H, O-CH), 3.79 (dd, $J=11.3$ and $3.4 \mathrm{~Hz}, 1 \mathrm{H}, \mathrm{O}-\underline{\mathrm{HCH}}), 3.65(\mathrm{dd}, J=11.3$ and $6.1 \mathrm{~Hz}, 1 \mathrm{H}, \mathrm{O}-\underline{\mathrm{HCH}}), 2.76-3.0\left(\mathrm{~m}, 4 \mathrm{H}, \mathrm{Te}-\mathrm{CH}_{2}\right.$ and $2 \mathrm{O}-\mathrm{H}), 2.07-2.17(\mathrm{~m}, 4 \mathrm{H}), 1.54-1.65(\mathrm{~m}, 4 \mathrm{H}) .{ }^{13} \mathrm{C}-\mathrm{NMR}\left(\mathrm{CDCl}_{3}, 100 \mathrm{MHz}\right) ; \delta(\mathrm{ppm}): 136.1,121.1$, $113.5,71.7,66.5,40.7,29.3,25.5,22.2,21.4,14.5$. MS: $m / z$ (rel. int.) 310 ( $\left.\mathrm{M}^{+}, 20.6\right), 235$ (6.8), 105 (100.0), 91 (43.0), 79 (54.0). HRMS: Calculated mass for $\mathrm{C}_{11} \mathrm{H}_{16} \mathrm{O}_{2} \mathrm{Te}:[\mathrm{M}+\mathrm{H}]^{+}$311.0291, found: 311.0273.

3-(4-tert-Buthylphenylethynyltellanyl)propane-1,2-diol 4c (Table 2, entry 3): Yield: $0.170 \mathrm{~g}$ (47\%); red oil. ${ }^{1} \mathrm{H}-\mathrm{NMR}\left(\mathrm{CDCl}_{3}, 400 \mathrm{MHz}\right) ; \delta$ (ppm): 7.29-7.35 (m, 4H, Ar-H), 4.03-4.09 (m, 1H, O-CH), 3.80 (dd, $J=11.4$ and $3.1 \mathrm{~Hz}, 1 \mathrm{H}, \mathrm{O}-\underline{\mathrm{HCH}}), 3.64(\mathrm{dd}, J=11.4$ and $6.2 \mathrm{~Hz}, 1 \mathrm{H}, \mathrm{O}-\underline{\mathrm{HCH}}), 3.03-3.30\left(\mathrm{~m}, 4 \mathrm{H}, \mathrm{Te}-\mathrm{CH}_{2}\right.$ and $2 \mathrm{O}-\mathrm{H}), 1.28\left(\mathrm{~s}, 9 \mathrm{H}, \mathrm{C}-\mathrm{CH}_{3}\right) .{ }^{13} \mathrm{C}-\mathrm{NMR}\left(\mathrm{CDCl}_{3}, 100 \mathrm{MHz}\right) ; \delta(\mathrm{ppm}): 151.8,131.6,125.2,120.4,111.3$, 71.6, 66.5, 44.3, 34.7, 31.1, 14.7. MS: $m / z$ (rel. int.) $362\left(\mathrm{M}^{+}, 20.2\right), 288$ (11.4), 143 (100), 57 (98.2), 41 (44.1). HRMS: Calculated mass for $\mathrm{C}_{15} \mathrm{H}_{20} \mathrm{O}_{2} \mathrm{Te}:[\mathrm{M}+\mathrm{H}]^{+}$363.0604, found: 363.0583 .

\subsection{Procedure for the Synthesis of the 3-Iodo-2-(2,2-dimethyl-1,3-dioxolanylmethyl)selenanylbenzo[b]furan 5}

To a solution of 2,2-dimethyl-1,3-dioxolanylmethyl(2-methoxyphenylethynyl)selane $3 \mathbf{f}(0.25 \mathrm{mmol})$ in $\mathrm{CH}_{2} \mathrm{Cl}_{2}(2.0 \mathrm{~mL})$ a solution of $\mathrm{I}_{2}(0.28 \mathrm{mmol})$ in $\mathrm{CH}_{2} \mathrm{Cl}_{2}(3.0 \mathrm{~mL})$ was added. The reaction mixture was stirred for $1 \mathrm{~h}$ at room temperature. Then, saturated aqueous $\mathrm{Na}_{2} \mathrm{~S}_{2} \mathrm{O}_{3}$ was added to remove the excess of $\mathrm{I}_{2}$. The mixture was then extracted with ethyl acetate $(3 \times 10 \mathrm{~mL})$ and the organic phase was separated, dried over $\mathrm{MgSO}_{4}$ and concentrated under vacuum. The product was isolated by column chromatography using hexanes/ethyl acetate as eluent.

Analytical Data of Product 5

3-Iodo-2-(2,2-dimethyl-1,3-dioxolanylmethyl)selenanylbenzo[b]furan 5 (Scheme 2): Yield: $0.093 \mathrm{~g}$ (85\%); yellow oil; ${ }^{1} \mathrm{H}-\mathrm{NMR}\left(\mathrm{CDCl}_{3}, 400 \mathrm{MHz}\right) ; \delta(\mathrm{ppm}): 7.44-7.47(\mathrm{~m}, 1 \mathrm{H}, \mathrm{Ar}-\mathrm{H}), 7.28-7.37$ (m, 3H, Ar-H), 4.36-4.42 (m, 1H, O-CH), $4.18(\mathrm{dd}, J=8.5$ and $6.0 \mathrm{~Hz}, 1 \mathrm{H}, \mathrm{O}-\underline{\mathrm{HCH}}), 3.79(\mathrm{dd}, J=8.5$ and $6.0 \mathrm{~Hz}, 1 \mathrm{H}$, $\mathrm{O}-\underline{\mathrm{HCH}}), 3.31(\mathrm{dd}, J=12.3$ and $5.2 \mathrm{~Hz}, 1 \mathrm{H}, \mathrm{Se}-\underline{\mathrm{HCH}}), 3.11(\mathrm{dd}, J=12.3$ and $7.8 \mathrm{~Hz}, 1 \mathrm{H}, \mathrm{Se}-\underline{\mathrm{HCH}}), 1.45(\mathrm{~s}$, $\left.3 \mathrm{H}, \mathrm{C}-\mathrm{CH}_{3}\right), 1.36\left(\mathrm{~s}, 3 \mathrm{H}, \mathrm{C}-\mathrm{CH}_{3}\right) .{ }^{13} \mathrm{C}-\mathrm{NMR}\left(\mathrm{CDCl}_{3}, 100 \mathrm{MHz}\right) ; \delta(\mathrm{ppm}): 156.7,146.6,131.3,125.6,123.6$, 121.2, 111.0; 109.8; 75.8, 75.4; 69.1; 30.8, 27.0; 25.5. MS: $m / z$ (rel. int.) $438\left(\mathrm{M}^{+}, 23.4\right), 168$ (18.2), 115 (35.3), 101 (21.5), 57 (100.0). HRMS: Calculated mass for $\mathrm{C}_{14} \mathrm{H}_{15} \mathrm{IO}_{3}$ Se: $\left[\mathrm{M}+\mathrm{NH}_{4}\right]^{+}$455.9575, found: 455.9577 .

\section{Conclusions}

In summary, we developed a new and general protocol to prepare glycerol-derived organoselanyl and organotellanyl alkynes using tosyl solketal. Eight organoselanyl and five organotellanyl alkynes were obtained in good yields and short reaction times, when compared to previously described 
procedures. Some of the organotellanyl alkynes were deprotected using Dowex 50WX8- $\left(\mathrm{H}^{+}\right)$to give new water-soluble 3-organotellanylpropane-1,2-diols.

Supplementary Materials: Supplementary materials are available at http://www.mdpi.com/1420-3049/22/3/ $391 /$ s1.

Acknowledgments: The authors thank FAPERGS, CNPq and CAPES for financial support. CNPq is also acknowledged for the fellowship for E.J.L., D.A. and G.P. This manuscript is part of the scientific activity of the international multidisciplinary "SeS Redox and Catalysis" network.

Author Contributions: E.J.L. and G.P. conceived and designed the experiments; E.L.B., G.S. and L.K.S. performed the experiments; D.A., R.F.S., L.B., F.M., E.J.L. and G.P. analyzed the data and wrote the paper.

Conflicts of Interest: The authors declared no conflict of interest.

\section{References}

1. Wirth, T. Organoselenium Chemistry—Modern Developments in Organic Synthesis; Topics in Current Chemistry; Springer: Heidelberg, Germany, 2000; Volume 208.

2. Zeni, G.; Lüdtke, D.S.; Panatieri, R.B.; Braga, A.L. Vinylic Tellurides: From Preparation to Their Applicability in Organic Synthesis. Chem. Rev. 2006, 106, 1032-1076. [CrossRef] [PubMed]

3. Perin, G.; Lenardão, E.J.; Jacob, R.G.; Panatieri, R.B. Synthesis of Vinyl Selenides. Chem. Rev. 2009, 109, 1277-1301. [CrossRef] [PubMed]

4. Menezes, P.H.; Zeni, G. Vinyl Selenides in Patai's Chemistry of Functional Groups; John Wiley \& Sons: New York, NY, USA, 2011.

5. Palomba, M.; Bagnoli, L.; Marini, F.; Santi, C.; Sancineto, L. Recent advances in the chemistry of vinylchalcogenides. Phosphorus Sulfur Silicon Relat. Elem. 2016, 191, 235-244. [CrossRef]

6. Nogueira, C.W.; Rocha, J.B.T. Organoselenium and organotellurium compounds: Toxicology and pharmacology. Chem. Rev. 2004, 104, 6255-6285. [CrossRef] [PubMed]

7. Goswami, S.; Hazra, A.; Chakrabarty, R.; Fun, H.-K. Recognition of carboxylate anions and carboxylic acids by selenium-based new chromogenic fluorescent sensor: A remarkable fluorescence enhancement of hindered carboxylates. Org. Lett. 2009, 11, 4350-4353. [CrossRef] [PubMed]

8. Rampon, D.S.; Rodembush, F.S.; Schneider, J.M.F.M.; Bechtold, I.H.; Gonçalves, P.F.B.; Merlo, A.A.; Schneider, P.H. Novel selenoesters fluorescent liquid crystalline exhibiting a rich phase polymorphism. J. Mater. Chem. 2010, 20, 715-722. [CrossRef]

9. Samb, I.; Bell, J.; Toullec, P.Y.; Michelet, V.; Leray, I. Fluorescent phosphane selenide as efficient mercury chemodosimeter. Org. Lett. 2011, 13, 1182-1185. [CrossRef] [PubMed]

10. Mugesh, G.; du Mont, W.-W.; Sies, H. Chemistry of biologically important synthetic organoselenium compounds. Chem. Rev. 2001, 101, 2125-2179. [CrossRef] [PubMed]

11. Santoro, S.; Azeredo, J.B.; Nascimento, V.; Sancineto, L.; Braga, A.L.; Santi, C. The green side of the moon: Ecofriendly aspects of organoselenium chemistry. RSC Adv. 2014, 4, 31521-31535. [CrossRef]

12. Santi, C. Organoselenium Chemistry between Synthesis and Biochemistry, 1st ed.; Bentham eBooks: Perugia, Italy, 2014.

13. Barcellos, A.M.; Abenante, L.; Sarro, M.T.; Leo, I.D.; Lenardão, E.J.; Perin, G.; Santi, C. New prospective for redox modulation mediated by organoselenium and organotellurium compounds. Curr. Org. Chem. 2017, 21, 1-18. [CrossRef]

14. Pérez-Balado, C.; Markó, I.E. 1-Iodo-1-selenoalkenes as versatile alkene 1,1-dianion equivalents. Novel connective approach towards the tetrahydropyran subunit of polycavernoside A. Tetrahedron 2006, 62, 2331-2349. [CrossRef]

15. Liu, C.-R.; Yang, F.-L.; Jin, Y.-Z.; Ma, X.-T.; Cheng, D.-J.; Li, N.; Tian, S.-K. Catalytic regioselective synthesis of structurally diverse indene derivatives from $n$-benzylic sulfonamides and disubstituted alkynes. Org. Lett. 2010, 12, 3832-3835. [CrossRef] [PubMed]

16. Mitamura, T.; Ogawa, A. Palladium-catalyzed alkynylselenation of acetylenedicarboxylates leading to enyne selenides and application to synthesis of multisubstituted aryl selenides. Tetrahedron Lett. 2010, 51, 3538-3541. [CrossRef] 
17. Okoronkwo, A.E.; Godoi, B.; Schumacher, R.F.; Neto, J.S.S.; Luchese, C.; Prigol, M.; Nogueira, C.W.; Zeni, G. $\mathrm{Csp}^{3}$-tellurium copper cross-coupling: Synthesis of alkynyl tellurides a novel class of antidepressive-like compounds. Tetrahedron Lett. 2009, 50, 909-915. [CrossRef]

18. Savegnago, L.; Borges, V.C.; Alves, D.; Jesse, C.R.; Rocha, J.B.T.; Nogueira, C.W. Evaluation of antioxidant activity and potential toxicity of 1-buthyltelurenyl-2-methylthioheptene. Life Sci. 2006, 79, 1546-1552. [CrossRef] [PubMed]

19. Ávila, D.S.; Gubert, P.; Palma, A.; Colle, D.; Alves, D.; Nogueira, C.W.; Rocha, J.B.T.; Soares, F.A.A. An organotellurium compound with antioxidant activity against excitotoxic agents without neurotoxic effects in brain of rats. Brain Res. Bull. 2008, 76, 114-123. [CrossRef] [PubMed]

20. Manarin, F.; Roehrs, J.A.; Gay, R.M.; Brandão, R.; Menezes, P.H.; Nogueira, C.W.; Zeni, G. Electrophilic cyclization of 2-chalcogenealkynylanisoles: Versatile access to 2-chalcogen-benzo[b]furans. J. Org. Chem. 2009, 74, 2153-2162. [CrossRef] [PubMed]

21. Mantovani, A.C.; Back, D.F.; Zeni, G. Chalcogenoalkynes: Precursors for the regioselective preparation of 2-chalcogeno-1-halonaphthalenes through [4+2] cycloaddition. Eur. J. Org. Chem. 2012, 4574-4579. [CrossRef]

22. Lara, R.G.; Borges, E.L.; Lenardão, E.J.; Alves, D.; Jacob, R.G.; Perin, G. Addition of thiols to phenylselenoalkynes using KF/alumina under solvent-free conditions. J. Braz. Chem. Soc. 2010, 21, 2125-2129. [CrossRef]

23. Perin, G.; Borges, E.L.; Alves, D. Highly stereoselective method to prepare bis-phenylchalcogen alkenes via addition of chalcogenolate to phenylseleno alkynes. Tetrahedron Lett. 2012, 53, 2066-2069. [CrossRef]

24. Rampon, D.S.; Giovenardi, R.; Silva, T.L.; Rambo, R.S.; Merlo, A.A.; Schneider, P.H. Chalcogenoacetylenes obtained by indium(III) catalysis: Dual catalytic activation of diorgano dichalcogenides and $\mathrm{C}_{\mathrm{sp}}-\mathrm{H}$ bonds. Eur. J. Org. Chem. 2011, 7066-7070. [CrossRef]

25. Godoi, M.; Ricardo, E.W.; Frizon, T.E.; Rocha, M.S.T.; Singh, D.; Paixão, M.W.; Braga, A.L. An efficient synthesis of alkynyl selenides and tellurides from terminal acetylenes and diorganyl diselenides or ditellurides catalyzed by recyclable copper oxide nanopowder. Tetrahedron 2012, 68, 10426-10430. [CrossRef]

26. Ahammed, S.; Bhadra, S.; Kundu, D.; Sreedhar, B.; Ranu, B.C. An efficient and general procedure for the synthesis of alkynyl chalcogenides (selenides and tellurides) by alumina-supported $\mathrm{Cu}$ (II)-catalyzed reaction of alkynyl bromides and diphenyl dichalcogenides. Tetrahedron 2012, 68, 10542-10549. [CrossRef]

27. Movasssagh, B.; Yousefi, A.; Momeni, B.Z.; Heydari, S. A general and highly efficient protocol for the synthesis of chalcogenoacetylenes by copper(I)-terpyridine catalyst. Synlett 2014, 25, 1385-1390. [CrossRef]

28. Godoi, M.; Liz, D.G.; Ricardo, E.W.; Rocha, M.S.T.; Azeredo, J.B.; Braga, A.L. Magnetite $\left(\mathrm{Fe}_{3} \mathrm{O}_{4}\right)$ nanoparticles: An efficient and recoverable catalyst for the synthesis of alkynyl chalcogenides (selenides and tellurides) from terminal acetylenes and diorganyl dichalcogenides. Tetrahedron 2014, 70, 3349-3354. [CrossRef]

29. Mohammadi, E. Movassagh, B. Cryptand-22 as an efficient ligand for the copper-catalyzed cross-coupling reaction of diorgano dichalcogenides with terminal alkynes leading to the synthesis of alkynyl chalcogenides. Tetrahedron Lett. 2014, 55, 1613-1615. [CrossRef]

30. Alves, D.; Sachini, M.; Jacob, R.G.; Lenardão, E.J.; Contreira, M.E.; Savegnago, L.; Perin, G. Synthesis of (Z)-organylthioenynes using $\mathrm{KF} / \mathrm{Al}_{2} \mathrm{O}_{3}$ /solvent as recyclable system. Tetrahedron Lett. 2011, 52, 133-135. [CrossRef]

31. Len, C.; Luque, R. Continuous flow transformations of glycerol to valuable products: An overview. Sustain. Chem. Process. 2014, 2, 1-10. [CrossRef]

32. Mori, K. Pheromone synthesis. Part 253: Synthesis of the racemates and enantiomers of triglycerides of male Drosophila fruit flies with special emphasis on the preparation of enantiomerically pure 1-monoglycerides. Tetrahedron 2012, 68, 8441-8449. [CrossRef]

33. Manzo, E.; Ciavatta, M.L.; Pagano, D.; Fontana, A. An efficient and versatile chemical synthesis of bioactive glyco-glycerolipids. Tetrahedron Lett. 2012, 53, 879-881. [CrossRef]

34. Wu, W.; Li, R.; Malladi, S.S.; Warshakoon, H.J.; Kimbrell, M.R.; Amolins, M.W.; Ukani, R.; Datta, A.; David, S.A. Structure-activity relationships in toll-like receptor-2 agonistic diacylthioglycerol lipopeptides. J. Med. Chem. 2010, 53, 3198-3213. [CrossRef] [PubMed]

35. Goubert, M.; Canet, I.; Sinibaldi, M.-E. An expedient route to new spiroheterocycles: Synthesis and structural studies. Eur. J. Org. Chem. 2006, 4805-4812. [CrossRef] 
36. Oh, K.; Yamada, K.; Asami, T.; Yoshizawa, Y. Synthesis of novel brassinosteroid biosynthesis inhibitors based on the ketoconazole scaffold. Bioorg. Med. Chem. Lett. 2012, 22, 1625-1628. [CrossRef] [PubMed]

37. Gong, L.; Hirschfeld, D.; Tan, Y.-C.; Hogg, J.H.; Peltz, G.; Avnur, Z.; Dunten, P. Discovery of potent and bioavailable GSK-3 $\beta$ inhibitors. Bioorg. Med. Chem. Lett. 2010, 20, 1693-1696. [CrossRef] [PubMed]

38. Perin, G.; Borges, E.L.; Duarte, J.E.G.; Webber, R.; Jacob, R.G.; Lenardão, E.J. Glycerol as renewable resource in the synthesis of thioethers using $\mathrm{KF} / \mathrm{Al}_{2} \mathrm{O}_{3}$. Curr. Green. Chem. 2014, 1, 115-120. [CrossRef]

39. Nobre, P.C.; Borges, E.L.; Silva, C.M.; Casaril, A.M.; Martinez, D.M.; Lenardão, E.J.; Alves, D.; Savegnago, L.; Perin, G. Organochalcogen compounds from glycerol: Synthesis of new antioxidants. Bioorg. Med. Chem. 2014, 22, 6242-6249. [CrossRef] [PubMed]

40. Borges, E.L.; Peglow, T.J.; Silva, M.S.; Jacoby, C.G.; Schneider, P.H.; Lenardão, E.J.; Jacob, R.G.; Perin, G. Synthesis of enantiomerically pure bis(2,2-dimethyl-1,3-dioxolanylmethyl)chalcogenides and dichalcogenides. New J. Chem. 2016, 40, 2321-2326. [CrossRef]

41. Soares, L.K.; Silva, R.B.; Peglow, T.J.; Silva, M.S.; Jacob, R.G.; Alves, D.; Perin, G. Selective synthesis of vinyl- or alkynyl chalcogenides from glycerol and their water-soluble derivatives. ChemistrySelect 2016, 1, 2009-2013. [CrossRef]

Sample Availability: Samples of the compounds 2-5 are available from the authors.

(C) 2017 by the authors. Licensee MDPI, Basel, Switzerland. This article is an open access article distributed under the terms and conditions of the Creative Commons Attribution (CC BY) license (http:/ / creativecommons.org/licenses/by/4.0/). 